Relations industrielles

Industrial Relations

\title{
Manpower Programs in the Policy Mix, by Lloyd Ulman (ed.). Baltimore, The John Hopkins University Press, 1973, 166 pp.
}

\section{Jacques Mercier}

Volume 29, numéro 1, 1974

URI : https://id.erudit.org/iderudit/028492ar

DOI : https://doi.org/10.7202/028492ar

Aller au sommaire du numéro

Éditeur(s)

Département des relations industrielles de l'Université Laval

ISSN

0034-379X (imprimé)

1703-8138 (numérique)

Découvrir la revue

Citer ce compte rendu

Mercier, J. (1974). Compte rendu de [Manpower Programs in the Policy Mix, by Lloyd Ulman (ed.). Baltimore, The John Hopkins University Press, 1973, 166 pp.] Relations industrielles / Industrial Relations, 29(1), 229-230.

https://doi.org/10.7202/028492ar

Tous droits réservés (C) Département des relations industrielles de l'Université Laval, 1974
Ce document est protégé par la loi sur le droit d'auteur. L'utilisation des services d'Érudit (y compris la reproduction) est assujettie à sa politique d'utilisation que vous pouvez consulter en ligne.

https://apropos.erudit.org/fr/usagers/politique-dutilisation/ 
Le mérite de ce livre pourrait être de faire renaître un débat autour de ce thème et de reposer le problème du contrôle syndical obtenu par la négociation collective, de la bureaucratisation du syndicalisme et de son intégration.

\section{Gilles LAFLAMME}

\section{Université Laval}

Manpower Programs in the Policy Mix, by Lloyd Ulman (ed.). Baltimore, The John Hopkins University Press, 1973, $166 \mathrm{pp}$.

Cet ouvrage présente cinq essais synthétiques sur les possibilités et le rốle de la politique de main-d'oeuvre au niveau macroéconomique. On y reprend le débat déjà amplement discuté sur le conflit d'arbitrage entre l'inflation et le chômage (courbe de Phillips). Les présentations de R. Gordon et de C. Holt reprennent en substance des points de vue qu'ils ont déjà soutenus dans des études récentes et qui portent sur l'analyse de facteurs structurels et frictionnels de l'emploi sur le marché du travail. La notion centrale de leurs thèses est celle de «segmentation» du marché du travail.

L'article de R. Meidner et R. Anderson à la fin du volume, sur la nature et l'impact des politiques sélectives de marché du travail pratiquées en Suède et qu'on retrouve dans le fameux modèle de Rehn, donne une illustration intéressante des actions sectorielles préconisées par R.A. Gordon, en tenant compte toutefois des différences contextuelles tant politiques et sociales qu'économiques. S. Levitan considère la portée des programmes actuels américains de main-d'oeuvre à déplacer la courbe de Phillips vers la gauche, tandis que L. Thurrow s'arrête sur les effets redistributifs de tels programmes.

Les discussions au sujet de la validité de la relation originale ainsi que des versions revues et corrigées de la Courbe de Phillips n'ont pas fini de soulever des divergences de point de vue. Bien qu'à prime abord il semble qu'on trouve des contrastes entre les divers auteurs, ceux-ci résultent davantage des conceptions sur les rapports entre le chômage et l'inflation et des problèmes envisagés.

Gordon et Holt fondent leurs observations sur une théorie «frictionnelle élargie du chômage.

Gordon offre une typologie du chômage basée sur les conditions de mobilité interne et externe propre à chaque segment, qui nie toute homogénéité du marché et en appelle à des mesures correctrices distinctes axées sur la réduction des taux de roulement et de la durée de la recherche d'emploi.

Ces considérations reposent toutefois sur un indice de dispersion du chômage qui n'a pour le moment qu'une valeur descriptive, tout comme le rapport vacances d'emplois/taux de chômage et salaire monétaire utilisé par Holt et ses collègues chez qui l'analyse porte sur le long terme et ne couvre partiellement qu'un aspect (cost-markup) de la théorie inflationniste des prix. Gordon constate un accroissement de la dispersion entre les taux de chômage par occupation ainsi que par sexe et âge, incidemment relié aux changements dans la composition de la main-d'oeuvre active. On ne peut toutefois $\mathrm{y}$ voir une relation entre l'inflation et le chômage. Holt introduit à cet effet un modèle empirique qui peut compléter l'indice de Gordon. Suivent certaines recommandations pertinentes pour l'avenir des politiques de maind'oeuvre.

Avec Thurrow, c'est par une critique acerbe des objectifs de croissance, de stabilisation et de plein emploi qu'est abordé le rôle de la politique de maind'oeuvre. Pour l'auteur de «Human Capital $\gg$, l'échec des politiques fiscales et monétaires relève principalement d'obstacles créés par l'émergence de pouvoirs institutionnels sur les marchés des produits comme des facteurs; les groupes défavorisés (pauvres, victimes de conflits sociaux) absorbent continuellement ces échecs. Aussi, la politique de main-d'oeuvre, si elle se veut un moyen d'atteindre l'équité sociale, ne doit surtout pas reprendre les mêmes chemins.

Certains resteront surpris de la façon un peu trop expéditive avec laquelle l'au- 
teur élimine la participation de la politique de main-d'oeuvre aux objectifs économiques. Toutefois, cette ligne de pensée recouvre assez bien l'idée prédominante des décisions en main-d'oeuvre depuis le passage de l'Economic Opportunity Act en 1964.

Dans l'esprit du lecteur, il peut y avoir confusion entre politique de maind'oeuvre et formation professionnelle, Thurrow ne faisant pas la distinction; ensuite, lorsqu'il parle du revenu «gagné » comme élément d'équité, il n'est pas certain que le revenu relatif se soit vraiment accru: des effets d'augmentation dans la concurrence pour des classes d'emplois dits «secondaires 》, des effets régressifs dans l'imposition de ces faibles revenus «non-imposables》 sous le couvert de taxes à la consommation, ne sont pas étrangers au phénomène des écarts de revenus. Enfin, après les tentatives de définition du chômage selon les causes, les effets ou encore les mesures correctives, Thurrow nous présente une distinction originale basée sur la valeur sociale accordée aux coûts de réduction du chômage : c'est sans doute la définition la plus franche qu'on en ait donnée jusqu'à présent.

C'est également, comme nous le disions plus haut, ce qui différencie son point de vue de ceux de Gordon et Holt: ceux-ci s'en tiennent à une vision générale du fonctionnement du marché du travail, ce dernier considère des choix sociaux.

Le texte de Levitan est le seul qui s'appuie sur une définition du rôle et des buts de la politique de main-d'oeuvre américaine et qui traite de ses limites pratiques dont peut-être la plus importante est l'insignifiance relative des montants qui y sont consacrés, ce qui lui fait conclure : «If manpower programs ease the unemployment-inflation tradeoff, so much the better $\gg$ (p. 116). Enfin. en plus de l'intérêt que peut présenter pour un public nord-américain l'expérience suédoise, un pays qui a délibérément opté pour la recherche du pleinemploi, on trouvera dans l'article de Meidner et Anderson des considérations qui élargissent les concepts de chômage et de participation à l'emploi.
Tout au long du livre, on y sent la relativité des explications et des positions, mais il n'en demeure pas moins que Manpower Programs in the Policy Mix est sans doute le meilleur ouvrage paru à date sur les relations entre les programmes de main-d'oeuvre et la position de la Courbe de Phillips. La portée des études des quatre auteurs américains reste toutefois fort limitée et pour le moment spéculative, tant qu'on n'aura pas approfondi davantage par la recherche empirique les relations proposées. Elle éclaire cependant d'un angle nouveau la théorie du marché du travail sous-jacente aux actions prises par nos gouvernants en main-d'oeuvre, du moins théoriquement. L'ouvrage sera un point de repère précieux pour ceux qui s'intéressent ou sont impliqués dans la politique de main-d'oeuvre et l'analyse du marché du travail.

Jacques MERCIER

Université Laval

Industry and Humanity, par William Lyon Mackenzie King, Toronto, The University of Toronto Press, 1973, $354 \mathrm{pp}$.

Pour la plupart d'entre nous, William Lyon Mackenzie King a été premier ministre du Canada de 1921 à 1926 et l'on ignore ou oublie souvent qu'il a d'abord fait carrière comme expert en relations industrielles et consultant auprès du gouvernement canadien et de plusieurs grandes corporations américaines. Et c'est à la fin de sa carrière à la Fondation Rockfeller et au moment où il allait faire ses débuts comme leader du parti libéral national qu'il écrit Industry and Humanity dont la publication originale, pour être plus précis, remonte à 1918 .

Replacer Industry and Humanity dans le contexte de l'industrie d'avant et pendant la première guerre, et l'évolution de la pensée de King ne rendrait certes pas hommage à David Jay Bercuson qui signe l'introduction de cette réédition, réalisée par les Presses de l'Université de Toronto. Dans cette présentation re- 